\title{
Trends in adult current asthma prevalence and contributing risk factors in the United States by state: $2000-2009$
}

\author{
Xingyou Zhang ${ }^{1 *}$, Teresa Morrison-Carpenter ${ }^{2}$, James B Holt ${ }^{1}$ and David B Callahan ${ }^{2}$
}

\begin{abstract}
Background: Current asthma prevalence among adults in the United States has reached historically high levels. Although national-level estimates indicate that asthma prevalence among adults increased by 33\% from 2000 to 2009, state-specific temporal trends of current asthma prevalence and their contributing risk factors have not been explored.

Methods: We used 2000-2009 Behavioral Risk Factor Surveillance System data from all 50 states and the District of Columbia (D.C.) to estimate state-specific current asthma prevalence by 2-year periods (2000-2001, 2002-2003, 2004-2005, 2006-2007, 2008-2009). We fitted a series of four logistic-regression models for each state to evaluate whether there was a statistically significant linear change in the current asthma prevalence over time, accounting for sociodemographic factors, smoking status, and weight status (using body mass index as the indicator).

Results: During 2000-2009, current asthma prevalence increased in all 50 states and D.C., with significant increases in 46/50 (92\%) states and D.C. After accounting for weight status in the model series with sociodemographic factors, and smoking status, 10 states ( $A R, A Z, I A, I L, K S, M E, M T, U T, W V$, and $W Y$ ) that had previously shown a significant increase did not show a significant increase in current asthma prevalence.

Conclusions: There was a significant increasing trend in state-specific current asthma prevalence among adults from 2000 to 2009 in most states in the United States. Obesity prevalence appears to contribute to increased current asthma prevalence in some states.
\end{abstract}

Keywords: Current asthma, Smoking, Obesity, Trend, The United States

\section{Background}

Current asthma prevalence among adults in the United States has reached historically high levels. In 2009, 8.4\% of U.S. adults (19.5 million persons) reported asthma compared with $7.2 \%$ (14.7 million persons) in 2000 [1,2]. Although national-level estimates indicate that asthma prevalence among adults relatively increased by $33 \%(4.8$ million persons) from 2000 to 2009 (almost 500,000 adults per year) [1,2], state specific temporal tends in asthma prevalence and their contributing risk factors have not been explored.

To determine the prevalence of current asthma in the United States each year, the Centers for Disease Control

\footnotetext{
*Correspondence: gyx8@cdc.gov

'Division of Population Health, National Center for Chronic Disease Prevention and Health Promotion, Centers for Disease Control and Prevention, 4770 Buford Highway, Mailstop F78, Atlanta 30341, GA, USA Full list of author information is available at the end of the article
}

and Prevention (CDC) uses national and state-based surveillance systems. Since the late 1970s, the National Health Interview Survey (NHIS), a national-based survey of households from all 50 states and the District of Columbia (D.C.), has provided a national estimate of asthma prevalence by population-based characteristics each year in the United States [3]. However, a systematic state-based survey, the Behavioral Risk Factor Surveillance System (BRFSS), provides state-level estimates of asthma prevalence by population-based characteristics each year.

Estimates of current asthma prevalence from both the national- (NHIS) and the state-based (BRFSS) surveys show that current asthma prevalence among adults is increasing [1,2,4-6] and continuing to vary by certain sociodemographic, behavioral, and geographic factors [7-12]. More specifically, asthma prevalence can vary by

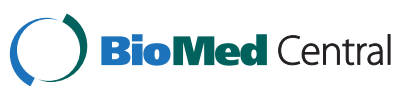


certain modifiable (e.g., weight and smoking status) [13-25] and non-modifiable (e.g., age and race status) risk factors [7-11,26-30]; however, the extent to which these risk factors contribute to increasing prevalence at the state level over time has not been analyzed or determined. Identifying state-specific changes in asthma prevalence and determining how known risk factors for asthma contribute to changes in prevalence over time are important for public health planning and for generating hypotheses for asthma prevention and control.

\section{Methods}

\section{Sample population}

We used cross-sectional data from the 2000-2009 BRFSS to estimate state-specific prevalence in current asthma and evaluate the influence of risk factors on current asthma prevalence [31]. BRFSS is a state-based random-digit dialing telephone survey that annually collects information on health conditions and risk behaviors of adults 18 years and older noninstitutionalized U.S. population in all 50 states, D.C., and U.S. territories. It uses a disproportionate stratified sample (DSS) design that commonly divides telephone numbers into two strata for sampling and also disproportionately samples smaller geographically defined populations of interest within a state. Further details on BRFSS survey methods and data are available at www.cdc.gov/brfss/index.htm.

\section{Measures}

The outcome measure is current asthma status (yes or no). In the 2000 BRFSS, all respondents were asked, "Did a doctor ever tell you that you had asthma?" From 2001 to 2009, the BRFSS question was changed and all respondents were asked, "Have you ever been told by a doctor, nurse, or other health professional that you had asthma?" From 2000 to 2009, if the respondent confirmed that they had been told they had asthma, they then were asked, "Do you still have asthma?" We defined current asthma as an affirmative response to both questions. To reduce the annual variability in survey measures [32], we combined two consecutive survey years and categorized "year of survey" into five 2-year periods: 2000-2001, 2002-2003, 2004-2005, 2006-2007, and 2008-2009.

To evaluate the effect of known risk factors on current asthma prevalence, we included BRFSS self-reported sociodemographic factors (age, sex, race/ethnicity, education, and household income) and modifiable behaviors (smoking status and weight status) associated with current asthma prevalence and available from BRFSS [31]. Age was categorized into six groups: 18-24, 25-34, $35-44,45-54,55-64$, and $\geq 65$ years. Race and ethnicity were categorized as non-Hispanic white, non-Hispanic black, Hispanic, and other. Education was categorized as less than high school, completion of high school, some college (including associate's degree), and a bachelor's degree or higher. Household income was categorized as: $<\$ 15,000, \$ 15,000-\$ 24,999, \$ 25,000-\$ 49,999, \$ 50,000-$ $\$ 74,999, \geq \$ 75,000$, or missing. Missing income values were included as a separate category because $13 \%$ of records in the 2000-2009 BRFSS did not specify income values. We categorized smoking status into three groups: current smokers (respondents who had smoked at least 100 cigarettes in their entire life and currently smoke), previous smokers (respondents who had smoked at least 100 cigarettes in their entire life but no longer smoke), and nonsmokers (respondents who had never smoked or had smoked fewer than 100 cigarettes in their life). To classify a respondent's weight status, we used body mass index (BMI; calculated as respondent's reported weight in kilograms divided by respondent's reported height in meters squared). Using BMI, we categorized weight status into five groups: underweight (BMI $\left.<18.5 \mathrm{~kg} / \mathrm{m}^{2}\right)$, healthy weight $\left(18.5 \mathrm{~kg} / \mathrm{m}^{2} \leq \mathrm{BMI}<25.0 \mathrm{~kg} / \mathrm{m}^{2}\right)$, overweight $\left(25.0 \mathrm{~kg} / \mathrm{m}^{2} \leq \mathrm{BMI}<30.0 \mathrm{~kg} / \mathrm{m}^{2}\right)$, obese $(30.0 \mathrm{~kg} /$ $\mathrm{m}^{2} \leq \mathrm{BMI}<40.0 \mathrm{~kg} / \mathrm{m}^{2}$ ), and morbidly obese (BMI $\geq 40.0 \mathrm{~kg} / \mathrm{m}^{2}$ ) [33].

\section{Statistical analysis}

All statistical data analyses were conducted using SASCallable SUDAAN software (Version 10, Research Triangle Institute, NC). The BRFSS complex survey design structures were considered in all current asthma outcome estimates by state, year of survey, sociodemographic factors (sex, age, race/ethnicity, household income, and education), smoking status, and weight status; BRFSS final post-stratified sampling weights were used in all statistical data analyses. We reported weighted percentages with $95 \%$ confidence intervals (CIs) for all population or subpopulation groups for current asthma prevalence estimates. For all analyses, we considered a $\mathrm{P}$ value of $<0.05$ significant. We fitted a series of four logistic regression models to examine temporal changes in asthma prevalence from 2000 to 2009 for the United States, each state, and D.C. The first model (model I) included only the continuous variable of 2-year survey periods to evaluate the linear trend in the prevalence of current asthma over the time period from 2000-2001 to 2008-2009. The second model (model II) adjusted for sociodemographic factors (age, sex, race/ethnicity, education, and household income) to evaluate whether an increasing linear trend in current asthma prevalence still existed. The third model (model III) introduced smoking status to evaluate its impact on the linear trends in current asthma prevalence after adjusting for individual sociodemographic factors. The fourth model (model IV) introduced weight status and evaluated its effect on the linear trends in current 
asthma prevalence while controlling for individual sociodemographic factors and smoking status. We used the model-based predicted marginal prevalence ratios (PRs) and prevalence differences (PDs) and P values from their related $\mathrm{t}$-Test to assess the direction and significance of linear trends in current asthma prevalence after controlling for the temporal changes in population sociodemographics, smoking, and weight status within a state. The model-adjusted prevalence differences for survey periods 2000-2001 compared to 2008-2009 were used to evaluate the increasing magnitude of asthma prevalence [34].

\section{Results}

From 2000 to 2009, BRFSS collected records on 3,203,280 respondents from the 50 states and D.C. Of these respondents, 159,407 (5.0\%) were excluded from the analysis because values for asthma status or sociodemographic factors other than household income, smoking status, or weight status were missing; 3,043,873 respondents were included in the analysis. The average sample size per year from 2000 to 2009 was 304,387 (range: from 174,810 in 2000 to 411,406 in 2007) and per state was 6,000 (range: from 4,621 in WY to 16,112 in WA). Survey response rates varied by year and state; the median state survey response rate for this time period was a little more than 50\% (detailed at http:// www.cdc.gov/brfss/technical_infodata/quality.htm).

\section{Current asthma prevalence}

Table 1 provides a summary of current asthma prevalence and unadjusted and adjusted prevalence ratios by 2-year periods, sociodemographic factors, smoking status, and weight status. Current asthma prevalence relatively increased by $18.2 \%$ nationally from $7.2 \%$ during 2000-2001 to 8.5\% during 2008-2009. Population subgroups with asthma prevalence greater than the 20082009 national level of $8.5 \%$ include persons who are: female (9.8\%), aged 18-24 years (9.4\%), black, nonHispanics (9.3\%); persons with: less than high school education (9.3\%), household income $<\$ 15,000$ (11.6\%), household income $\$ 15,000-\$ 25,000$ (9.1\%); and persons who: currently smoke (9.2\%), are obese $(10.2 \%)$, or are morbidly obese (18.2\%). Population subgroups with prevalence ratio (PR) estimates $>1.5$ for current asthma include persons: who are female (PR: 1.69, 95\% CI: 1.661.72 , compared to males); have household incomes $<\$ 15,000$ (PR: 1.58, 95\% CI: 1.53-1.64, compared to $\geq \$ 75,000$ ); are obese (PR: $1.63,95 \%$ CI: 1.60-1.67, compared to healthy weight); or are morbidly obese (PR: 2.60, 95\% CI: 2.52-2.68, compared to healthy weight).

\section{Trends in current asthma prevalence}

All 50 states and D.C. showed an increase in current asthma unadjusted prevalence from 2000-2001 to
2008-2009; and over the 10-year period, current asthma prevalence increased by $>2.0$ percentage points in 9 states (AK, DC, HI, LA, NH, NJ, OK, PA, and SD) and by 1.5-2.0 percentage points in 12 states (DE, KY, MD, MI, NM, NY, OH, OR, TN, VA, VT, and WI) (Figure 1 and Table 2).

In the unadjusted analysis comparing two time periods of current asthma prevalence, 2000-2001 to 2008-2009, $80 \%(40 / 50)$ of states and D.C. showed significant increases. Only ten states (AR, AZ, CO, IA, MN, MT, ND, $\mathrm{NV}$, UT, and WV) showed increases in current asthma prevalence that were not statistically significant (Figure 2 and Table 3 [model I]). After controlling for survey year and changes in population sociodemographics, 46 states, including 6 of the 10 states (AR, AZ, IA, ND, UT, and WV) that showed insignificant increases in the unadjusted model (Figure 2 and [model I]), and D.C. showed a significant increase in current asthma prevalence in 2008-2009 compared to 2000-2001 (Figure 2 and Table 3 [model II]). When we added smoking status to the model with survey year and sociodemographic factors, MT shows a significant increase in current asthma prevalence and all other states keep unchanged in their linear trends of current asthma prevalence (Figure 2 and Table 3 [model III]) was observed except MT. However, when we added weight status to the model with survey year, sociodemographic factors, and smoking status, 10 states (AR, AZ, IA, IL, KS, ME, MTUT, WV , and WY,) that had previously shown a significant increasing trend showed no significant increasing linear trend in current asthma prevalence from 2000 to 2009 (Figure 2 and Table 3 [model IV]).

\section{Discussion}

Our findings indicate that current asthma prevalence among adults increased significantly from 2000-2001 to 2008-2009 for the majority of states (40/50) and for the U.S. as a whole. Our findings also suggest that place of residence, sociodemographic factors, and modifiable behaviors such as obesity might have contributed to those increases.

We considered population-based sociodemographic factors in the analysis because asthma prevalence is known to vary by certain population subgroups in the United States. Consistent with previous studies [8-12], asthma prevalence was higher among women than men and among non-Hispanic black than non-Hispanic white persons. Asthma was more prevalent among persons with less than a high school education than among persons with a bachelor degree, and also more prevalent among persons with an annual household income $<\$ 15,000$ than among persons with a household income $\geq \$ 75,000$. Over the last century, the demographic changes for the nation in general reveal a decrease in 
Table 1 U.S. adult current asthma prevalence and prevalence ratios (PR) by 2000-2009 BRFSS sample characteristics

\begin{tabular}{|c|c|c|c|c|c|}
\hline Characteristics & $\begin{array}{l}\text { Subpopulation } \\
\text { Groups }\end{array}$ & $\begin{array}{l}\text { Sample size } \\
n=3,043,873\end{array}$ & $\begin{array}{l}\text { Prevalence* } \\
\%\left(95 \% \mathrm{Cl}^{\dagger}\right)\end{array}$ & $\begin{array}{l}\text { Unadjusted } \\
\text { PR }\left(95 \% \mathrm{Cl}^{\dagger}\right)\end{array}$ & $\begin{array}{l}\text { Adjusted }^{\#} \\
\text { PR }\left(95 \% \mathrm{Cl}^{\dagger}\right)\end{array}$ \\
\hline 2-Year & $2000-2001$ & 374,137 & $7.21(7.07,7.35)$ & Reference & \\
\hline \multirow[t]{4}{*}{ Period } & 2002-2003 & 486,368 & $7.60(7.47,7.74)$ & $1.04(1.04,1.05)$ & $1.04(1.04,1.05)$ \\
\hline & 2004-2005 & 629,633 & $7.99(7.86,8.12)$ & $1.09(1.07,1.10)$ & $1.09(1.07,1.10)$ \\
\hline & 2006-2007 & 748,536 & $8.23(8.10,8.37)$ & $1.13(1.11,1.15)$ & $1.13(1.11,1.15)$ \\
\hline & 2008-2009 & 805,199 & $8.52(8.40,8.65)$ & $1.18(1.15,1.20)$ & $1.18(1.15,1.20)$ \\
\hline \multirow[t]{2}{*}{ Sex } & Male & $1,176,599$ & $5.90(5.82,5.99)$ & Reference & \\
\hline & Female & $1,867,274$ & $9.83(9.75,9.92)$ & $1.67(1.64,1.69)$ & $1.69(1.66,1.72)$ \\
\hline Age, & $18-24$ & 159,715 & $9.36(9.11,9.62)$ & Reference & \\
\hline \multirow[t]{5}{*}{ in years } & $25-34$ & 388,151 & $7.65(7.51,7.80)$ & $0.82(0.79,0.84)$ & $0.79(0.76,0.82)$ \\
\hline & $35-44$ & 537,390 & $7.44(7.32,7.56)$ & $0.79(0.77,0.82)$ & $0.74(0.72,0.76)$ \\
\hline & $45-54$ & 623,754 & $7.98(7.85,8.10)$ & $0.85(0.83,0.88)$ & $0.75(0.73,0.78)$ \\
\hline & $55-64$ & 560,588 & $8.43(8.29,8.57)$ & $0.90(0.87,0.93)$ & $0.75(0.73,0.77)$ \\
\hline & $\geq 65$ & 774,275 & $7.28(7.17,7.40)$ & $0.78(0.75,0.80)$ & $0.63(0.61,0.65)$ \\
\hline Race and & White $(\mathrm{NH})^{* *}$ & $2,451,608$ & $8.09(8.03,8.16)$ & Reference & \\
\hline \multirow[t]{3}{*}{ Ethnicity } & Black (NH) & 234,974 & $9.26(9.04,9.49)$ & $1.15(1.12,1.17)$ & $0.96(0.94,0.99)$ \\
\hline & Hispanic & 188,189 & $5.86(5.66,6.06)$ & $0.72(0.70,0.75)$ & $0.64(0.62,0.67)$ \\
\hline & Others & 169,102 & $8.37(8.09,8.66)$ & $1.03(1.00,1.07)$ & $1.05(1.01,1.08)$ \\
\hline \multirow[t]{4}{*}{ Education } & $<$ High school & 310,985 & $9.26(9.06,9.46)$ & $1.32(1.28,1.35)$ & $1.12(1.09,1.16)$ \\
\hline & High school & 936,827 & $7.85(7.74,7.96)$ & $1.12(1.10,1.14)$ & $0.93(0.91,0.95)$ \\
\hline & Some college & 815,293 & $8.48(8.36,8.60)$ & $1.21(1.18,1.23)$ & $1.02(1.00,1.04)$ \\
\hline & >Bachelor degree & 980,768 & $7.03(6.93,7.12)$ & Reference & \\
\hline Household & $<\$ 15,000$ & 301,442 & $11.6(11.4,11.9)$ & $1.74(1.69,1.79)$ & $1.58(1.53,1.64)$ \\
\hline \multirow[t]{5}{*}{ Income } & $\$ 15 k^{\# \#} \sim<\$ 25 k$ & 474,354 & $9.06(8.90,9.23)$ & $1.35(1.32,1.39)$ & $1.27(1.24,1.31)$ \\
\hline & $\$ 25 k \sim<\$ 50 k$ & 811,140 & $7.54(7.42,7.65)$ & $1.13(1.10,1.15)$ & $1.07(1.05,1.10)$ \\
\hline & $\$ 50 \mathrm{k} \sim<\$ 75 \mathrm{k}$ & 449,348 & $7.05(6.91,7.20)$ & $1.05(1.03,1.08)$ & $1.01(0.98,1.04)$ \\
\hline & $\geq \$ 75 \mathrm{k}$ & 611,531 & $6.69(6.57,6.81)$ & Reference & \\
\hline & Unknown & 396,058 & $7.99(7.81,8.16)$ & $1.19(1.16,1.23)$ & $1.15(1.11,1.18)$ \\
\hline Smoking & Never & $1,595,769$ & $7.23(7.15,7.31)$ & Reference & \\
\hline \multirow[t]{2}{*}{ Status } & Former & 853,780 & $8.41(8.29,8.52)$ & $1.16(1.14,1.18)$ & $1.24(1.22,1.26)$ \\
\hline & Current & 594,324 & $9.21(9.07,9.35)$ & $1.27(1.25,1.3)$ & $1.26(1.24,1.29)$ \\
\hline Weight & Underweight & 50,089 & $8.70(8.22,9.20)$ & $1.29(1.22,1.37)$ & $1.10(1.04,1.17)$ \\
\hline \multirow[t]{4}{*}{ Status } & Healthy weight & $1,063,457$ & $6.75(6.66,6.85)$ & Reference & \\
\hline & Overweight & $1,064,475$ & $6.91(6.81,7.00)$ & $1.02(1.00,1.04)$ & $1.18(1.15,1.20)$ \\
\hline & Obese & 639,896 & $10.2(10.0,10.3)$ & $1.51(1.48,1.54)$ & $1.63(1.60,1.67)$ \\
\hline & Morbidly obese & 96,785 & $18.2(17.7,18.7)$ & $2.69(2.62,2.78)$ & $2.60(2.52,2.68)$ \\
\hline
\end{tabular}

*Weighted prevalence for complex survey design; ${ }^{*}$ Adjusted by logistic regression for all factors listed; ${ }^{\dagger} \mathrm{Cl}=\mathrm{Confidence} \mathrm{Interval;}$ ${ }^{* *} \mathrm{NH}=$ Non-Hispanic $;{ }^{\# \#} \mathrm{k}=$ thousand.

the men-to-women ratio and an increase in minority populations [35]. These findings, coupled with the pattern of sociodemographic factors among adults with asthma, suggest that the increase in asthma prevalence among adults might be due, in part, to changes in population subgroups. The number of population subgroups with higher proportions of persons with asthma (e.g., women, non-Hispanic black persons) has been increasing at a higher rate than has subgroups with relatively lower proportions of persons with asthma (e.g., men, non-Hispanic white persons). When these changes were accounted for in the analysis, the number of states with a significant linear increasing trends in current asthma prevalence increased from $40(80 \%)$ to 46 (92\%) states, 

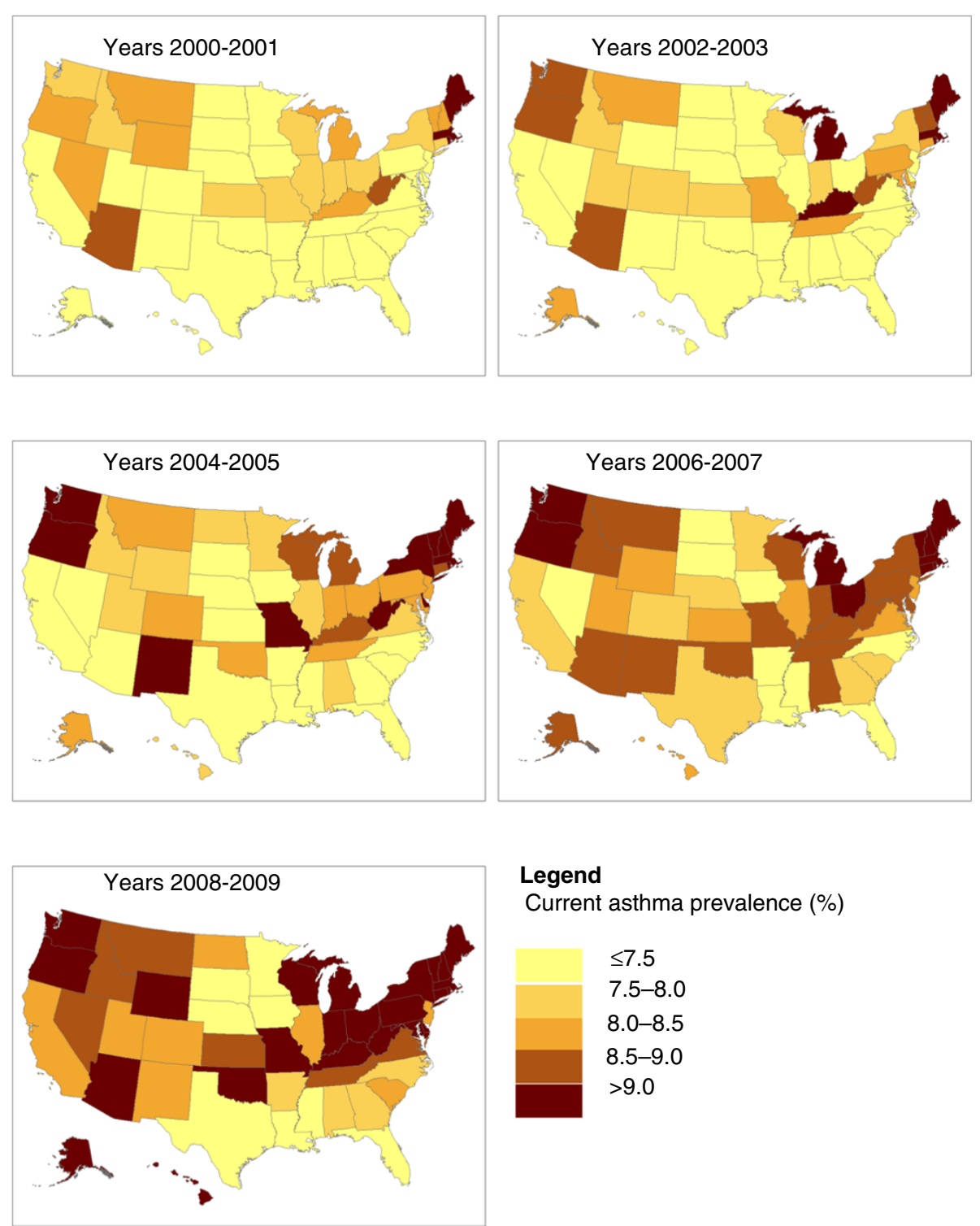

Legend

Current asthma prevalence (\%)

\section{$\leq 7.5$}

$7.5-8.0$

$8.0-8.5$

$8.5-9.0$

$>9.0$

Figure 1 Current asthma unadjusted prevalence by state.

including 6 of the10 states (AR, AZ, IA, ND, UT, and WV) and D.C. that had insignificant increasing linear trends when state-specific changes in sociodemographic subgroups were not considered.

In this analysis, the number of states with significant increases in current asthma prevalence did not change when smoking status was added to the model with survey year and sociodemographic factors. It seems that the positive public health effects of tobacco control have become overshadowed by other factors contributing to the increase in current asthma prevalence in the last decade. However, when weight status was considered with survey year, sociodemographic factors, and smoking status, 10 states (AK, IL, KS, ME, WY, AR, AZ, IA, UT, and WV) that had previously shown a significant increase in current asthma prevalence when considering those variables showed no significant increase in prevalence. These findings suggest that the high prevalence of asthma among obese $(10.2 \%, 95 \%$ CI $10.0-10.3)$ and morbidly obese $(18.2 \%$, 95\% CI $17.7-18.7)$ persons, coupled with the increasing prevalence of obese persons in the United States [36], could contribute to observed increases in adult asthma prevalence in some states. An analysis comparing two cycles of data from the National Health and Nutrition Examination Survey (NHANES 2001-2002 and 2003-2004) similarly showed that adult asthma prevalence is increasing with greater prevalence among obese and morbidly obese adults [7].

Modifiable factors such as obesity and smoking have been associated with the development of asthma 
Table 2 Average 2-year current asthma unadjusted prevalence among adults by state, BRFSS, 2000-2009

\begin{tabular}{|c|c|c|c|c|c|}
\hline \multirow[t]{3}{*}{ State } & \multicolumn{5}{|c|}{ 2-year period } \\
\hline & 2000-2001 & $2002-2003$ & 2004-2005 & 2006-2007 & 2008-2009 \\
\hline & $\%\left(\mathrm{se}^{*}\right)$ & $\%\left(s e^{*}\right)$ & $\%\left(\mathrm{se}^{*}\right)$ & $\%\left(\mathrm{se}^{*}\right)$ & $\%\left(s^{*}\right)$ \\
\hline US & $7.21(0.07)$ & $7.60(0.07)$ & $7.99(0.07)$ & $8.23(0.07)$ & $8.52(0.07)$ \\
\hline AK & $7.17(0.58)$ & $8.30(0.60)$ & $8.48(0.58)$ & $8.64(0.57)$ & $9.23(0.67)$ \\
\hline $\mathrm{AL}$ & $6.23(0.38)$ & $7.33(0.38)$ & $7.91(0.40)$ & $8.74(0.44)$ & $7.71(0.37)$ \\
\hline$A R$ & $6.81(0.39)$ & $7.46(0.35)$ & $7.45(0.33)$ & $7.24(0.31)$ & $7.93(0.43)$ \\
\hline$A Z$ & $8.66(0.71)$ & $8.65(0.54)$ & $7.36(0.47)$ & $8.85(0.53)$ & $10.2(0.67)$ \\
\hline CA & $7.23(0.32)$ & $7.44(0.33)$ & $7.46(0.32)$ & $7.64(0.31)$ & $8.20(0.23)$ \\
\hline $\mathrm{CO}$ & $7.36(0.45)$ & $7.95(0.35)$ & $8.48(0.33)$ & $7.86(0.27)$ & $8.18(0.26)$ \\
\hline$C T$ & $7.91(0.31)$ & $8.44(0.32)$ & $8.97(0.35)$ & $9.27(0.34)$ & $9.14(0.43)$ \\
\hline DC & $7.62(0.52)$ & $8.60(0.56)$ & $9.10(0.50)$ & $9.65(0.45)$ & $10.0(0.51)$ \\
\hline $\mathrm{DE}$ & $7.37(0.46)$ & $7.49(0.40)$ & $9.11(0.47)$ & $8.71(0.46)$ & $9.19(0.55)$ \\
\hline $\mathrm{FL}$ & $5.71(0.27)$ & $6.29(0.32)$ & $7.03(0.29)$ & $6.71(0.23)$ & $6.97(0.33)$ \\
\hline GA & $6.76(0.36)$ & $7.23(0.34)$ & $7.35(0.38)$ & $7.84(0.30)$ & $7.82(0.45)$ \\
\hline $\mathrm{HI}$ & $7.36(0.38)$ & $6.27(0.31)$ & $7.53(0.45)$ & $8.02(0.35)$ & 9.49 (0.38) \\
\hline IA & $6.58(0.34)$ & $6.36(0.34)$ & $6.95(0.31)$ & $6.74(0.31)$ & $7.20(0.32)$ \\
\hline ID & $7.83(0.32)$ & $7.83(0.33)$ & $7.64(0.32)$ & $8.91(0.39)$ & $8.62(0.39)$ \\
\hline $\mathrm{IL}$ & $7.89(0.41)$ & $7.29(0.28)$ & $7.74(0.34)$ & $8.32(0.36)$ & $8.47(0.36)$ \\
\hline IN & $7.85(0.37)$ & $7.78(0.28)$ & $8.28(0.29)$ & $8.52(0.33)$ & $9.18(0.38)$ \\
\hline KS & $7.98(0.34)$ & $7.60(0.32)$ & $7.18(0.24)$ & $8.34(0.30)$ & $8.64(0.27)$ \\
\hline KY & $8.01(0.31)$ & $9.67(0.38)$ & $8.53(0.37)$ & $8.55(0.37)$ & $9.87(0.39)$ \\
\hline LA & $5.07(0.25)$ & $6.10(0.28)$ & $6.08(0.31)$ & $6.12(0.29)$ & $7.22(0.32)$ \\
\hline MA & $9.03(0.28)$ & $9.44(0.31)$ & $9.68(0.32)$ & $9.89(0.27)$ & $10.3(0.29)$ \\
\hline $\mathrm{MD}$ & $7.27(0.39)$ & $8.13(0.39)$ & $8.13(0.33)$ & $8.66(0.32)$ & $9.25(0.34)$ \\
\hline ME & $9.12(0.49)$ & $9.99(0.50)$ & $9.85(0.44)$ & $9.96(0.38)$ & $10.6(0.35)$ \\
\hline $\mathrm{Ml}$ & $8.19(0.42)$ & $9.04(0.37)$ & $8.67(0.29)$ & $9.50(0.35)$ & $9.98(0.32)$ \\
\hline MN & $6.89(0.35)$ & $7.15(0.34)$ & $7.98(0.41)$ & $7.72(0.38)$ & $7.22(0.37)$ \\
\hline $\mathrm{MO}$ & $7.78(0.40)$ & $8.27(0.41)$ & $9.05(0.42)$ & $8.62(0.43)$ & $9.01(0.46)$ \\
\hline MS & $6.14(0.41)$ & $6.54(0.32)$ & $7.09(0.32)$ & $6.74(0.29)$ & $7.34(0.28)$ \\
\hline MT & $8.07(0.47)$ & $8.41(0.43)$ & $8.30(0.37)$ & $8.72(0.37)$ & $8.89(0.36)$ \\
\hline NC & $6.69(0.37)$ & $6.78(0.32)$ & $7.02(0.20)$ & $7.31(0.23)$ & $7.71(0.26)$ \\
\hline ND & $7.20(0.44)$ & $7.18(0.38)$ & $7.54(0.39)$ & $7.33(0.39)$ & $8.42(0.41)$ \\
\hline $\mathrm{NE}$ & $6.22(0.37)$ & $7.16(0.32)$ & $6.83(0.28)$ & $7.76(0.38)$ & 7.39 (0.33) \\
\hline $\mathrm{NH}$ & $8.42(0.44)$ & $8.59(0.33)$ & $10.3(0.36)$ & $10.0(0.36)$ & $10.4(0.39)$ \\
\hline NJ & $6.19(0.29)$ & $7.44(0.41)$ & $8.09(0.22)$ & $8.14(0.30)$ & $8.23(0.28)$ \\
\hline NM & $6.95(0.35)$ & $7.36(0.32)$ & $9.12(0.34)$ & $8.52(0.35)$ & $8.49(0.35)$ \\
\hline NV & $8.31(0.56)$ & $7.16(0.47)$ & $7.08(0.50)$ & $7.39(0.45)$ & $8.71(0.55)$ \\
\hline NY & $7.62(0.37)$ & $7.81(0.31)$ & $9.18(0.32)$ & $8.71(0.33)$ & $9.36(0.35)$ \\
\hline $\mathrm{OH}$ & $7.96(0.43)$ & $7.20(0.35)$ & $8.17(0.41)$ & $9.37(0.48)$ & $9.83(0.33)$ \\
\hline OK & $6.67(0.31)$ & $7.34(0.26)$ & $8.41(0.28)$ & $8.80(0.30)$ & $9.48(0.33)$ \\
\hline OR & $8.21(0.38)$ & $8.94(0.38)$ & $9.96(0.31)$ & $9.85(0.40)$ & $9.90(0.45)$ \\
\hline PA & $7.01(0.34)$ & $8.10(0.32)$ & $8.45(0.30)$ & $8.97(0.35)$ & $9.20(0.32)$ \\
\hline $\mathrm{Rl}$ & $9.01(0.39)$ & $9.18(0.38)$ & $10.2(0.43)$ & $10.2(0.45)$ & $10.4(0.42)$ \\
\hline SC & $6.63(0.36)$ & $5.94(0.31)$ & $7.07(0.26)$ & $7.61(0.28)$ & $8.02(0.34)$ \\
\hline
\end{tabular}


Table 2 Average 2-year current asthma unadjusted prevalence among adults by state, BRFSS, 2000-2009 (Continued)

\begin{tabular}{llllll}
\hline SD & $5.43(0.26)$ & $6.63(0.32)$ & $7.04(0.30)$ & $7.39(0.34)$ & $7.47(0.35)$ \\
TN & $7.06(0.38)$ & $8.11(0.42)$ & $8.26(0.40)$ & $8.57(0.43)$ & $7.70(0.38)$ \\
TX & $6.29(0.26)$ & $7.00(0.28)$ & $7.02(0.28)$ & $8.57(0.43)$ \\
UT & $7.42(0.44)$ & $7.64(0.42)$ & $7.96(0.35)$ & $8.35(0.38)$ & $8.95(0.29)$ \\
VA & $6.83(0.44)$ & $7.40(0.36)$ & $7.94(0.36)$ & $8.21(0.41)$ & $8.19(0.35)$ \\
VT & $8.02(0.35)$ & $8.59(0.37)$ & $9.09(0.32)$ & $9.46(0.34)$ & $9.90(0.51)$ \\
WA & $7.93(0.34)$ & $8.98(0.29)$ & $9.18(0.19)$ & $9.12(0.19)$ & $9.10(0.20)$ \\
WI & $7.77(0.41)$ & $7.99(0.36)$ & $8.89(0.37)$ & $8.95(0.43)$ & $9.53(0.48)$ \\
WV & $8.89(0.43)$ & $8.61(0.38)$ & $9.66(0.41)$ & $8.79(0.41)$ & $9.24(0.38)$ \\
WY & $8.47(0.43)$ & $7.37(0.35)$ & $7.76(0.33)$ & $8.45(0.34)$ & $9.12(0.34)$ \\
Median & $7.37(0.46)$ & $7.60(0.32)$ & $8.13(0.33)$ & $8.55(0.37)$ & $8.89(0.36)$ \\
\hline
\end{tabular}

se $=$ standard error.

[13-20,22-25], decline in lung function, severity of asthma symptoms, and diminished response to steroid medication [37-42]. Although the pathophysiologic mechanisms remain unclear, genetic studies indicate that the relationship between asthma and obesity might be explained by independent and combined biologic pathways [42]. An international population-based cohort study that collected DNA samples from 9,167 participants found an independent association between obesity and asthma (OR 2.4, 95\% CI 1.7-3.2) and a stronger, combined effect (OR 6.1, 95\% CI 2.5-14.4) among nonatopic obese persons [42]. An increased risk of asthma onset and exacerbation has been found also among current or former smokers, especially among women and nonatopic adults [43]. In one study among nonatopic adults, $20 \%$ of adult-onset asthma was attributed to current smoking, suggesting that a large proportion of adult-onset asthma could be prevented by smoking cessation [43]. A population-based, nested, case-control study of adults (aged 21-51 years) in Sweden reported an increased risk for adult-onset asthma associated with noninfectious rhinitis that occurred before asthma onset (OR 5.4, 95\% CI 4.0-7.2), especially among smoking nonatopic adults (OR 9.1, 95\% CI 5.3-15.4), and among persons who smoked before asthma onset (OR 1.5, 95\% CI 1.1-21) [44].
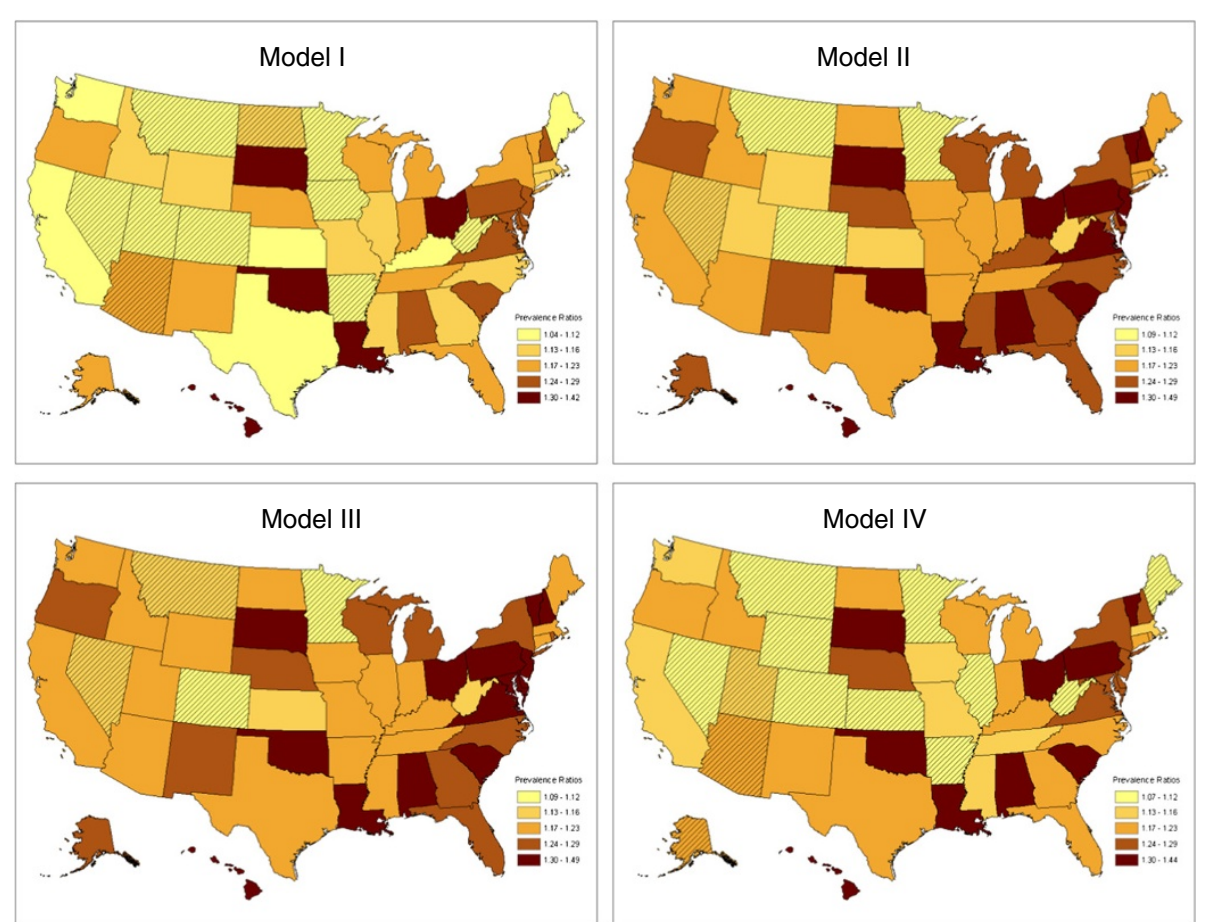

Figure 2 Current asthma prevalence ratios (PRs) for 2008-2009 vs 2000-2001. 
Table 3 Current asthma prevalence ratios (PR) for 2008-2009 vs 2000-2001 and significance by state

\begin{tabular}{|c|c|c|c|c|}
\hline State & Model I & Model II & Model III & Model IV \\
\hline$\overline{\mathrm{AL}}$ & $1.26(1.11,1.42)^{* * *}$ & $1.37(1.20,1.55)^{* * *}$ & $1.37(1.21,1.55)^{* * *}$ & $1.31(1.15,1.49)^{* * *}$ \\
\hline AK & $1.23(1.03,1.48)^{*}$ & $1.28(1.06,1.54)^{* *}$ & $1.28(1.06,1.54)^{* *}$ & $1.21(1.00,1.46)^{*}$ \\
\hline$A Z$ & $1.19(0.98,1.43)$ & $1.23(1.02,1.48)^{*}$ & $1.23(1.02,1.48)^{*}$ & $1.20(0.99,1.45)$ \\
\hline AR & $1.12(0.98,1.27)$ & $1.18(1.04,1.35)^{*}$ & $1.18(1.03,1.35)^{*}$ & $1.12(0.98,1.29)$ \\
\hline CA & $1.12(1.02,1.23)^{*}$ & $1.19(1.08,1.31)^{* * *}$ & $1.20(1.09,1.32)^{* * *}$ & $1.15(1.05,1.27)^{* *}$ \\
\hline $\mathrm{CO}$ & $1.08(0.97,1.20)$ & $1.11(0.99,1.24)$ & $1.11(1.00,1.24)$ & $1.11(0.99,1.25)$ \\
\hline$C T$ & $1.16(1.05,1.29)^{* *}$ & $1.23(1.11,1.36)^{* * *}$ & $1.23(1 \cdot 11,1.36)^{* * *}$ & $1.18(1.06,1.32)^{* *}$ \\
\hline DE & $1.26(1.09,1.46)^{* *}$ & $1.35(1.16,1.56)^{* * *}$ & $1.35(1.17,1.56)^{* * *}$ & $1.25(1.07,1.45)^{* *}$ \\
\hline DC & $1.30(1.12,1.50)^{* * *}$ & $1.38(1.20,1.60)^{* * *}$ & $1.39(1.20,1.61)^{* * *}$ & $1.32(1.14,1.53)^{* * *}$ \\
\hline $\mathrm{FL}$ & $1.19(1.06,1.33)^{* *}$ & $1.24(1.11,1.39)^{* * *}$ & $1.25(1.11,1.39)^{* * *}$ & $1.19(1.06,1.34)^{* *}$ \\
\hline GA & $1.16(1.02,1.32)^{*}$ & $1.24(1.08,1.42)^{* *}$ & $1.24(1.09,1.42)^{* *}$ & $1.17(1.02,1.35)^{*}$ \\
\hline $\mathrm{HI}$ & $1.37(1.22,1.55)^{* * *}$ & $1.43(1.27,1.61)^{* * *}$ & $1.43(1.27,1.62)^{* * *}$ & $1.34(1 \cdot 18,1.51)^{* * *}$ \\
\hline ID & $1.14(1 \cdot 02,1 \cdot 27)^{*}$ & $1.20(1.08,1.34)^{* *}$ & $1.21(1.09,1.35)^{* * *}$ & $1.18(1.05,1.32)^{* *}$ \\
\hline IL & $1.13(1.01,1.27)^{*}$ & $1.17(1.04,1.31)^{* *}$ & $1.18(1.05,1 \cdot 32)^{* *}$ & $1.12(1.00,1.26)$ \\
\hline IN & $1.18(1.06,1.31)^{* *}$ & $1.21(1.09,1.35)^{* * *}$ & $1.22(1.09,1.36)^{* * * *}$ & $1.17(1.05,1.31)^{* *}$ \\
\hline IA & $1.10(0.98,1.24)$ & $1.19(1.05,1.34)^{* *}$ & $1.19(1.05,1.34)^{* *}$ & $1.13(1.00,1.28)^{*}$ \\
\hline KS & $1.11(1.01,1.22)^{*}$ & $1.14(1.04,1.26)^{* *}$ & $1.15(1.04,1.26)^{* *}$ & $1.10(1.00,1.22)$ \\
\hline KY & $1.12(1.02,1.24)^{*}$ & $1.24(1.12,1.37)^{* * *}$ & $1.23(1 \cdot 12,1 \cdot 37)^{* * *}$ & $1 \cdot 22(1 \cdot 10,1 \cdot 35)^{* * *}$ \\
\hline LA & $1.33(1.18,1.49)^{* * *}$ & $1.49(1.32,1.67)^{* * *}$ & $1.49(1.32,1.67)^{* * *}$ & $1.44(1.27,1.63)^{* * *}$ \\
\hline ME & $1.12(1.01,1.25)^{*}$ & $1.19(1.06,1.32)^{* *}$ & $1.19(1.06,1.33)^{* *}$ & $1.11(0.99,1.25)$ \\
\hline MD & $1.24(1.11,1.39)^{* * *}$ & $1.29(1.16,1.45)^{* * *}$ & $1.30(1.17,1.45)^{* * *}$ & $1.24(1.11,1.39)^{* * *}$ \\
\hline MA & $1.13(1.05,1.21)^{* *}$ & $1.17(1.08,1.26)^{* * *}$ & $1.18(1.09,1.27)^{* * *}$ & $1.14(1.06,1.23)^{* * *}$ \\
\hline $\mathrm{Ml}$ & $1.19(1.08,1.32)^{* * *}$ & $1.24(1 \cdot 12,1 \cdot 37)^{* * *}$ & $1.25(1 \cdot 12,1 \cdot 38)^{* * *}$ & $1.19(1.07,1.32)^{* *}$ \\
\hline MN & $1.07(0.94,1.20)$ & $1.09(0.96,1.23)$ & $1.09(0.96,1.23)$ & $1.07(0.94,1.21)$ \\
\hline MS & $1.16(1.03,1.32)^{*}$ & $1.24(1.09,1.40)^{* *}$ & $1.23(1.08,1.40)^{* *}$ & $1.16(1.02,1.33)^{*}$ \\
\hline $\mathrm{MO}$ & $1.14(1.01,1.29)^{*}$ & $1.22(1.07,1.38)^{* *}$ & $1.22(1.07,1.38)^{* *}$ & $1.16(1.02,1.32)^{*}$ \\
\hline MT & $1.10(0.97,1.24)$ & $1.12(0.99,1.27)$ & $1.13(1.00,1.27)$ & $1.09(0.96,1.23)$ \\
\hline NE & $1.18(1.04,1.33)^{* *}$ & $1.26(1.11,1.42)^{* * *}$ & $1.26(1.11,1.42)^{* * * *}$ & $1.24(1.09,1.40)^{* *}$ \\
\hline NV & $1.08(0.91,1.28)$ & $1.16(0.98,1.37)$ & $1.16(0.98,1.38)$ & $1.11(0.94,1.32)$ \\
\hline $\mathrm{NH}$ & $1.25(1.13,1.39)^{* * *}$ & $1.31(1.18,1.46)^{* * *}$ & $1.32(1.18,1.46)^{* * *}$ & $1.26(1.13,1.40)^{* * *}$ \\
\hline NJ & $1.28(1.16,1.42)^{* * *}$ & $1.31(1.19,1.45)^{* * *}$ & $1.32(1.19,1.46)^{* * *}$ & $1.27(1.14,1.41)^{* * *}$ \\
\hline NM & $1.23(1.10,1.36)^{* * *}$ & $1.26(1.13,1.40)^{* * *}$ & $1.26(1.13,1.41)^{* * * *}$ & $1.22(1.09,1.36)^{* * *}$ \\
\hline NY & $1.23(1.11,1.36)^{* * *}$ & $1.27(1.15,1.41)^{* * *}$ & $1.28(1 \cdot 15,1.42)^{* * *}$ & $1.24(1 \cdot 12,1 \cdot 38)^{* * *}$ \\
\hline NC & $1.16(1.04,1.29)^{* *}$ & $1.28(1.15,1.43)^{* * *}$ & $1.29(1.15,1.43)^{* * * *}$ & $1.19(1.06,1.33)^{* *}$ \\
\hline ND & $1.15(1.00,1.32)$ & $1.21(1.05,1.39)^{* *}$ & $1.21(1.06,1.39)^{* *}$ & $1.17(1.01,1.35)^{*}$ \\
\hline $\mathrm{OH}$ & $1.32(1.18,1.48)^{* * *}$ & $1.38(1.23,1.55)^{* * *}$ & $1.38(1.23,1.55)^{* * *}$ & $1.32(1.17,1.49)^{* * *}$ \\
\hline OK & $1.42(1.29,1.56)^{* * *}$ & $1.45(1.32,1.60)^{* * *}$ & $1.43(1.30,1.58)^{* * * *}$ & $1.36(1.23,1.51)^{* * *}$ \\
\hline OR & $1.20(1.07,1.33)^{* *}$ & $1.26(1.13,1.41)^{* * *}$ & $1.27(1.14,1.42)^{* * *}$ & $1 \cdot 23(1 \cdot 10,1 \cdot 37)^{* * *}$ \\
\hline PA & $1.29(1.16,1.42)^{* * *}$ & $1.34(1.21,1.48)^{* * *}$ & $1.34(1.22,1.48)^{* * *}$ & $1.30(1 \cdot 18,1.44)^{* * *}$ \\
\hline $\mathrm{Rl}$ & $1.16(1.05,1.29)^{* *}$ & $1.22(1.10,1.36)^{* * *}$ & $1.24(1.11,1.37)^{* * * *}$ & $1.18(1.06,1.31)^{* *}$ \\
\hline SC & $1.29(1.14,1.46)^{* * *}$ & $1.38(1.23,1.56)^{* * *}$ & $1.39(1.23,1.56)^{* * * *}$ & $1.35(1.19,1.53)^{* * *}$ \\
\hline SD & $1.32(1.18,1.49)^{* * *}$ & $1.41(1.25,1.58)^{* * *}$ & $1.41(1.25,1.58)^{* * *}$ & $1.37(1.21,1.54)^{* * *}$ \\
\hline TN & $1.18(1.04,1.34)^{* *}$ & $1.20(1.06,1.36)^{* *}$ & $1.19(1.05,1.36)^{* *}$ & $1.16(1.01,1.32)^{*}$ \\
\hline TX & $1.12(1.01,1.24)^{*}$ & $1.19(1.08,1.32)^{* * *}$ & $1.20(1.08,1.32)^{* * *}$ & $1.17(1.05,1.29)^{* *}$ \\
\hline
\end{tabular}


Table 3 Current asthma prevalence ratios (PR) for 2008-2009 vs 2000-2001 and significance by state (Continued)

\begin{tabular}{lllll}
\hline UT & $1.12(0.99,1.26)$ & $1.16(1.03,1.32)^{*}$ & $1.17(1.04,1.33)^{*}$ & $1.13(1.00,1.29)$ \\
VT & $1.23(1.11,1.35)^{* * *}$ & $1.33(1.20,1.46)^{* * *}$ & $1.33(1.20,1.46)^{* * *}$ & $1.30(1.18,1.44)^{* * *}$ \\
VA & $1.24(1.07,1.43)^{* *}$ & $1.31(1.14,1.52)^{* * *}$ & $1.32(1.14,1.53)^{* * *}$ & $1.27(1.10,1.48)^{* *}$ \\
WA & $1.11(1.03,1.20)^{* *}$ & $1.19(1.10,1.29)^{* * *}$ & $1.20(1.11,1.30)^{* * *}$ & $1.15(1.06,1.24)^{* * *}$ \\
WV & $1.04(0.93,1.16)$ & $1.16(1.04,1.30)^{* *}$ & $1.16(1.04,1.30)^{* *}$ & $1.09(0.98,1.23)$ \\
WI & $1.23(1.08,1.40)^{* *}$ & $1.28(1.13,1.45)^{* * *}$ & $1.28(1.13,1.46)^{* * *}$ & $1.22(1.07,1.39)^{* *}$ \\
WY & $1.13(1.01,1.27)^{*}$ & $1.16(1.04,1.30)^{*}$ & $1.17(1.04,1.31)^{* *}$ & $1.11(0.99,1.25)$ \\
US & $1.18(1.15,1.20)^{* * *}$ & $1.23(1.20,1.26)^{* * *}$ & $1.23(1.21,1.26)^{* * *}$ & $1.18(1.15,1.20)^{* * *}$ \\
\hline
\end{tabular}

* $p$ value less $0.05 ;{ }^{* *} p$ value less $0.01 ;{ }^{* * *} p$ value less 0.001 .

The factors accounted for in this analysis as well as other factors that could not be accounted for, such as changes in state public health policies, public and professional awareness of asthma symptoms, air pollution exposures, geographic locations (urban vs rural settings), and occupational exposures, might also be contributing to the observed increase in current asthma prevalence. Although the degree to which public and professional awareness has influenced asthma prevalence estimates could not be determined, the significant independent increase in current asthma prevalence by sociodemographic factors, smoking status, and weight status from this analysis suggests that the contribution might be relatively low, unless increased awareness and reporting of asthma symptoms follow similar patterns of variation. Substantial clinical and epidemiologic evidence suggest that indoor and outdoor air pollutants can increase asthma symptoms [45-53], and increasing evidence indicates that certain air pollutants can lead to asthma onset [54-59]. Although fewer studies on new onset asthma have been conducted among adults than children, several cohort studies have found that traffic-related local pollutants can contribute to the onset and manifestation of asthma in adults $[55,58,59]$. Also, a substantial proportion of adult-onset asthma has been attributed to occupational exposures [60-65]. In a population-based, 10-year prospective study of 6,837 adults (aged 20-44 years at study onset) from 13 countries that participated in the European Community Respiratory Health Surveys (ECRHS and ECRHS II) [66], Kogevinas et al. found large geographic variations in population attributable risk (PAR) for adult-onset asthma due to occupational exposures (PAR range 10-25\%); significant excess risk for nurses (relative risk [RR] 2.2, 95\% CI 1.3-4.0); and increased risk for participants who experienced an acute symptomatic inhalation event (e.g., fire, mixing cleaning products, or chemical spills) (RR 3.3, 95\% CI 1.0-11.1) [61]. Another populationbased cohort study of all employed adults aged 25-59 years in Finland from 1986 to 1998 found that a large proportion (400-500 new cases/million persons/year) of adult-onset asthma might have been attributable to occupational factors and, therefore, might have been prevented [64].

The results of this study are based on the analysis of a large representative population-based sample that was obtained by using standardized sampling methods and survey questions for all 50 states and D.C. over a 10-year period. However, three potential sampling frame errors that might have occurred due to nonresponse, noncoverage, and self-reported measures should be considered when interpreting the results. The median response rate for BRFSS each year was $\sim 50 \%$; however, asthma prevalence estimates in this study are similar to estimates in other studies with higher response rates, such as NHIS [67]. Noncoverage may occur because BRFSS does not survey institutionalized adults, the military, or residents without home telephones. We used BRFSS-weighted adjustments to minimize the effect of nonresponse and noncoverage. And, self-reported survey measurements may be less accurate than those based on physical measurements; however, a 1993 review of asthma questionnaires reported a sensitivity of $68 \%$ and specificity of $94 \%$ when self-reported asthma was compared to a clinical diagnosis of asthma [68].

\section{Conclusion}

From 2000 to 2009, current asthma prevalence among adults increased for all 50 states and D.C., with significant increases in 46/50 (92\%) states and D.C. After accounting for changes in population-based sociodemographic factors and smoking prevalence, the increasing prevalence of obese and morbidly obese persons in some states is associated with increasing prevalence of asthma, and if causal, could contribute to the increase.

\section{Next steps}

Advances in asthma prevention and control are likely to originate from an understanding of population and environmental characteristics associated with temporal and geographic variations in asthma occurrence across states. Therefore, asthma surveillance systems that track these 
changes should be developed, maintained, and expanded within states and territories to support local-level analyses of population-based characteristics and environmental factors. State-by-state analysis is useful not only for public health planning but also for generating and testing hypotheses to explain the etiologic risk factors for asthma. Further study to identify changes in prevalence and their contributing risk factors at the state, county, and community level should be pursued to improve our understanding of asthma etiology, to identify high-risk groups, and to support state- and local-level intervention strategies to prevent asthma occurrence and control asthma symptoms.

\section{Competing interests}

The authors declare that they have no competing interests.

\section{Authors' contributions}

All authors were responsible for the design and development of the study. $X Z$ and TMC equally involved in all phases of the study including designing of the study, data collection and data analysis, and write-up of the manuscript. JBH and DBC participated in its research design and final drafting of the manuscript. All authors read and approved the final manuscript.

\section{Disclaimer}

The findings and conclusions in this article are those of the authors and do not necessarily represent the official position of the Centers for Disease Control and Prevention.

\section{Author details}

'Division of Population Health, National Center for Chronic Disease Prevention and Health Promotion, Centers for Disease Control and Prevention, 4770 Buford Highway, Mailstop F78, Atlanta 30341, GA, USA. ${ }^{2}$ Centers for Disease Control and Prevention, National Center for Environmental Health, Air Pollution and Respiratory Health Branch, Atlanta, GA, USA.

Received: 28 June 2013 Accepted: 20 November 2013 Published: 10 December 2013

\section{References}

1. Centers for Disease Control and Prevention: 2009 Behavioral Risk Factor Surveillance System Data. Table C1, Adult self-reported current asthma prevalence rate (percent) and prevalence (number) by state or territory; 2010. Available at: http://www.cdc.gov/asthma/brfss/09/current/tableC1.htm Accessed May 5, 2011

2. Centers for Disease Control and Prevention: 2000 Behavioral Risk Factor Surveillance System Data. Table C1, Adult self-reported current asthma prevalence rate (percent) and prevalence (number) by state; 2010. Available at: http://www. cdc.gov/asthma/brfss/00/current/tableC1.htm Accessed May 5, 2011.

3. Centers for Disease Control and Prevention: National Health Interview Survey. About the National Health Interview Survey; 2011. Available at: http://www. cdc.gov/nchs/nhis/about_nhis.htm Accessed July 13, 2011.

4. Centers for Disease Control and Prevention: Behavioral Risk Factor Surveillance System (BRFSS) Prevalence Data. Background Information; 2011. Available at: http://www.cdc.gov/asthma/brfss/default.htm Accessed July 13, 2011.

5. Centers for Disease Control and Prevention: 2001 National Health Interview Survey Data. Table 4-1, Current asthma prevalence by age, United States; 2010. Available at: http://www.cdc.gov/asthma/nhis/01/data.htm Accessed December 22, 2010.

6. Centers for Disease Control and Prevention: 2009 National Health Interview Survey (NHIS) data: Table 4-1, Current asthma prevalence by age, United States: National Health Interview Survey, 2009; 2011. Available at: http://www. cdc.gov/asthma/nhis/09/data.htm Accessed July 13, 2011

7. McHugh MK, Symanski E, Pompeii LA, Delclos GL: Prevalence of asthma among adult females and males in the United States: results from the
National Health and Nutrition Examination Survey (NHANES), 2001-2004. J Asthma 2009, 46(8):759-766.

8. Centers for Disease Control and Prevention: Surveillance for asthmaUnited States, 1960-1995. MMWR Surveill Summ 1998, 47(SS01):1-28.

9. Mannino DM, Homa DM, Akinbami LJ, Moorman JE, Gwynn C, Redd SC: Surveillance for asthma-United States, 1980-1999. MMWR CDC Surveill Summ 2002, 51(SS01):1-13.

10. Rose D, Mannino DM, Leaderer BP: Asthma prevalence among US adults, 1998-2000: role of Puerto Rican ethnicity and behavioral and geographic factors. Am J Public Health 2006, 96:880-888.

11. Moorman JE, Rudd RA, Johnson CA, et al: National surveillance for asthmaUnited States, 1980-2004. MMWR Surveill Summ 2007, 56(SS08):1-13.

12. Centers for Disease Control and Prevention: Current asthma prevalenceUnited States, 2006-2008. MMWR 2011, 60(Suppl 01):S84-S86.

13. Chen Y, Rennie D, Cormier Y, Dosman J: Atopy, obesity and asthma in adults: the Humboldt Study. J Agromedicine 2009, 14(2):222-227.

14. Colilla S, Nicolae D, Pluzhnikov A, et al: Collaborative study for the genetics of asthma. Evidence for gene-environment interactions in a linkage study of asthma and smoking exposure. J Allergy Clin Immunol 2003, 111:840-846.

15. Dixon AE, Holguin F, Sood A, et al: An official American Thoracic Society workshop report: obesity and asthma. Proc Am Thorac Soc 2010, 7:325-335.

16. Gunnbjörnsdóttir Ml, Omenaas E, Gíslason T, et al: Obesity and nocturnal gastro-oesophageal reflux are related to onset of asthma and respiratory symptoms. Eur Respir J 2004, 24:116-121.

17. Guerra S, Wright AL, Morgan WJ, et al: Persistence of asthma symptoms during adolescence: role of obesity and age at onset of puberty. Am J Respir Crit Care Med 2004, 170:78-85.

18. Jaakkola MS, Piipari R, Jaakkola N, et al: Environmental tobacco smoke and adult-onset asthma: a population-based incident case-control study. Am J Public Health 2003, 93(12):2055-2060.

19. Jamrozik E, Knuiman MW, James A, et al: Risk factors for adult-onset asthma: a 14-year longitudinal study. Respirology 2009, 14(6):814-821.

20. Lugogo NL, Kraft M, Dixon AE: Does obesity produce a distinct asthma phenotype? J Appl Physiol 2010, 108(3):729-734.

21. Meyers DA, Postma DS, Stine OC, et al: Genome screen for asthma and bronchial hyperresponsiveness: interactions with passive smoke exposure. J Allergy Clin Immunol 2005, 115:1169-1175.

22. Nystad W, Meyer HE, Nafstad P, et al: Body mass index in relation to adult asthma among 135,000 Norwegian men and women. Am J Epidemiol 2004, 160:969-976.

23. Ramadas RA, Sadeghnejad A, Karmaus W, et al: Interleukin-1r antagonist gene and pre-natal smoke exposure are associated with childhood asthma. Eur Respir J 2007, 29:502-508.

24. Rönmark $E$, Andersson $C$, Nyström $L$, et al: Obesity increases the risk of incident asthma among adults. Eur Respir J 2005, 25:282-288.

25. Siroux V, Pin I, Oryszczyn MP, et al: Relationships of active smoking to asthma and asthma severity in the EGEA study. Eur Respir J 2000, 15:470-477.

26. on behalf of the ISAAC Steering Committee and the European Community Respiratory Health Survey, Pearce N, Sunyer J, Cheng S, et al: Comparison of asthma prevalence in the ISAAC and the ECRHS. ISAAC Steering Committee and the European Community Respiratory Health Survey. International Study of Asthma and Allergies in Childhood. Eur Resp J 2000, 16:420-426.

27. Sears MR, Justina MB, Greene M, et al: A longitudinal, population-based, cohort study of childhood asthma followed to adulthood. N Engl I Med 2003, 349:1414-1422.

28. Spahn JD, Covar R: Clinical assessment of asthma progression in children and adults. J Allergy Clin Immunol 2008, 121:548-557.

29. Stern DA, Morgan WJ, Halonen M, et al: Wheezing and bronchial hyperresponsiveness in early childhood as predictors of newly diagnosed asthma in early adulthood: a longitudinal birth-cohort study. Lancet 2008, 372:1058-1064.

30. Toelle BG, Xuan W, Peat JK, et al: Childhood factors that predict asthma in young adulthood. Eur Respir J 2004, 23:66-70.

31. Centers for Disease Control and Prevention: Behavioral Risk Factor Surveillance System. BRFSS Annual Survey Data. Survey Data and Documentation; 2011. Available at: http://www.cdc.gov/brfss/annual_data/annual_data.htm Accessed July 13,2011

32. Centers for Disease Control and Prevention: 2004 Behavioral Risk Factor Surveillance System Data. Prevalence and trends data, Hawaii - 2004; 2010 
Available at: http://apps.nccd.cdc.gov/brfss/page.asp?cat=AS\&yr=2004 \&state=HI\#AS Accessed May 31, 2011.

33. Centers for Disease Control and Prevention: Overweight and obesity; 2010. Available at: http://www.cdc.gov/obesity/defining.html Accessed February 24, 2011.

34. Bieler GS, Brown GG, Williams RL, et al: Estimating model-adjusted risks, risk differences, and risk ratios from complex survey data. Am J Epidemiol 2010, 171(5):618-623.

35. Hobbs F, Stoops N: U.S. Census Bureau, Census 2000 Special Reports, CENSR-4, Demographic trends in the $20^{\text {th }}$ century. Washington, D.C: U.S. Government Printing Office; 2002

36. Centers for Disease Control and Prevention: Vital signs: state-specific obesity prevalence among adults-United States, 2009. MMWR Morb Mortal Wkly Rep 2010, 59(30):951-955.

37. Peters-Golden M, Swern A, Bird SS, et al: Influence of body mass index on the response to asthma controller agents. Eur Respir J 2006, 27:495-503.

38. Rodrigo GJ, Plaza $\mathrm{V}$ : Body mass index and response to emergency department treatment in adults with severe asthma exacerbations: a prospective cohort study. Chest 2007, 132:1513-1519.

39. Sutherland ER, Goleva E, Strand M, et al: Body mass and glucocorticoid response in asthma. Am J Respir Crit Care Med 2008, 178:682-687.

40. Dixon AE, Shade DM, Cohen Rl, et al: Effect of obesity on clinical presentation and response to treatment in asthma. J Asthma 2006, 43:553-558.

41. Boulet LP, Franssen E: Influence of obesity on response to fluticasone with or without salmeterol in moderate asthma. Respir Med 2007, 101(11):2240-2247.

42. Castro-Giner F, Kogevinas M, Imboden M, et al: Joint effect of obesity and TNFA variability on asthma: two international cohort studies. Eur Respir $J$ 2009, 33(5):1003-1009.

43. McLeish AC, Zvolensky MJ: Asthma and cigarette smoking: a review of the empirical literature. J Asthma 2010, 47:345-361.

44. Torén K, Olin A-C, Hellgren J, et al: Rhinitis increase the risk for adult-onset asthma-a Swedish population-based case-control study (MAP-study). Respir Med 2002, 96:635-641.

45. Breysse PN, Diette GB, Matsul EC, Butz AM, Hansel NN, McCormack MC: Indoor air pollution and asthma in children. Proc AM Thorac Soc 2010, 7(2):102-106.

46. Gilliland FD: Outdoor air pollution, genetic susceptibility, and asthma management: opportunities for intervention to reduce the burden of asthma. Pediatrics 2009, 123(suppl3):S168-S173.

47. O'Connor GT, Neas L, Vaughn B, et al: Acute respiratory health effects of air pollution on children with asthma in US inner cities. J Allergy Clin Immunol 2008, 121(5):1133-1139.

48. McCreanor J, Cullinan P, Nieuwenhuijsen MJ, et al: Respiratory effects of exposure to diesel traffic in persons with asthma. N Engl J Med 2007, 357:2348-2358.

49. Gent JF, Triche EW, Holford TR, et al: Association of low-level ozone and fine particles with respiratory symptoms in children with asthma. JAMA 2003, 290(14):1859-1867.

50. Delfino RJ: Epidemiologic evidence for asthma and exposure to air toxics: linkages between occupational, indoor, and community air pollution research. Environ Health Perspect 2002, 110(suppl 4):573-589.

51. Gold DR, Damokosh Al, Pope CA III, et al: Particulate and ozone pollutant effects on the respiratory function of children in southwest Mexico City. Epidemiology 1999, 10(1):8-16.

52. Bascom R, Bromberg P, Costa D, et al: Health effects of outdoor air pollution, part 1. Committee of the Environmental and Occupational Health Assembly of the American Thoracic Society. Am J Respir Crit Care Med 1996, 153(1):3-50

53. Bascom R, Bromberg $P$, Costa $D$, et al: Health effects of outdoor air pollution, part 2. Committee of the Environmental and Occupational Health Assembly of the American Thoracic Society. Am J Respir Crit Care Med 1996, 153(2):477-498.

54. McConnell R, Islam T, Shankardass K, et al: Childhood incident asthma and traffic-related air pollution at home and school. Environ Health Perspect 2010, 118(7):1021-1026.

55. Jacquemin B, Sunyer J, Forsberg B, et al: Home outdoor NO2 and new onset of self-reported asthma in adults. Epidemiology 2009, 20(1):119-126.

56. Delfino RJ, Staimer N, Tjoa T, et al: Personal and ambient air pollution exposures and lung function decrements in children with asthma. Environ Health Perspect 2008, 116(4):550-558
57. Jerrett M, Shankardass K, Berhane K, et al: Traffic-related air pollution and asthma onset in children: a prospective cohort study with individual exposure measurement. Environ Health Perspect 2008, 116(10):1433-1438.

58. Künzli N, Bridevaux PO, Liu LJ, et al: Swiss cohort study on air pollution and lung diseases in adults. Traffic-related air pollution correlates with adult-onset asthma among never-smokers. Thorax 2009, 64(8):664-670.

59. Modig L, Torén K, Janson C, et al: Vehicle exhaust outside the home and onset of asthma among adults. Eur Respir J 2009, 33(6):1261-1267.

60. Dykewicz MS: Occupational asthma: current concepts in pathogenesis, diagnosis, and management. J Allergy Clin Immunol 2009, 123:519-528.

61. Kogevinas M, Zock JP, Jarvis D, et al: Exposure to substances in the workplace and new-onset asthma: an international prospective population-based study (ECHRS-II). Lancet 2007, 370:336-341.

62. Sama SR, Milton DK, Hunt PR, et al: Case-by-case assessment of adult-onset asthma attributable to occupational exposures among members of a health maintenance organization. J Occup Environ Med 2006, 48(4):400-407.

63. Balmes J, Becklake M, Blanc $P$, et al: American Thoracic Society statement: occupational contribution to the burden of airway disease. Am J Respir Crit Care Med 2003, 167:787-797.

64. Karjalainen A, Kurppa $K$, Martikainen $R$, et al: Work is related to a substantial portion of adult-onset asthma incidence in the Finnish population. Am J Respir Crit Care Med 2001, 164:565-568.

65. Milton DK, Solomon GM, Rosiello RA, et al: Risk and incidence of asthma attributable to occupational exposure among $\mathrm{HMO}$ members. Am J Ind Med 1998, 33:1-10.

66. European Community Respiratory Health Survey: Variations in the prevalence of respiratory symptoms, self-reported asthma attacks, and use of asthma medication in the European Community Respiratory Health Survey (ECRHS). Eur Respir J 1996, 9:687-695.

67. Centers for Disease Control and Prevention: Asthma surveillance dataNational Health Interview Survey (NHIS) data. Available at: http://www.cdc gov/asthma/nhis/default.htm Accessed July 18, 2011.

68. Torén K, Brisman J, Jarvholm B: Asthma and asthma-like symptoms in adults assessed by questionnaires: a literature review. Chest 1993, 104:600-608.

\section{doi:10.1186/1471-2458-13-1156}

Cite this article as: Zhang et al:: Trends in adult current asthma prevalence and contributing risk factors in the United States by state: 2000-2009. BMC Public Health 2013 13:1156.

\section{Submit your next manuscript to BioMed Central and take full advantage of:}

- Convenient online submission

- Thorough peer review

- No space constraints or color figure charges

- Immediate publication on acceptance

- Inclusion in PubMed, CAS, Scopus and Google Scholar

- Research which is freely available for redistribution 